\title{
Factors associated with injury severity among users of powered mobility devices
}

\section{Suk Won Choi' ${ }^{1}$ Jae-Hyug Woo², Sung Youl Hyun ${ }^{3}$, Jae Ho Jang' ${ }^{2}$, Woo Sung $\mathrm{Choi}^{2}$}

'Department of Emergency and Critical Care Medicine, Gachon University Gil Medical Center, Incheon, Korea

${ }^{2}$ Department of Emergency and Critical Care Medicine, Gachon University Gil Medical Center, Gachon University College of Medicine, Incheon, Korea

${ }^{3}$ Department of Traumatology, Gachon University Gil Medical Center, Gachon University College of Medicine, Incheon, Korea

Objective To examine the features of powered mobility device-related injuries and identify the predictors of injury severity in such settings.

Methods Emergency Department-based Injury In-depth Surveillance data from 2011 to 2018 were used in this retrospective study. Participants were assigned to the mild/moderate and severe groups based on their excess mortality ratio-adjusted injury severity score and their general injury-related factors and injury outcome-related factors were compared.

Results Of 407 patients, 298 (79.2\%) were assigned to the mild/moderate group and 109 (26.8\%) to the severe group. The severe group included a higher percentage of patients aged 70 years or older (43.0\% vs. 59.6\%, $P=0.003)$, injuries incurred in the daytime (72.6\% vs. $82.4 \%, P=0.044)$, injuries from traffic accidents and falls $(P=0.042)$, head injuries (38.6\% vs. 80.7\%, $P<0.001)$, torso injuries ( $16.8 \%$ vs. $32.1 \%, P=0.001)$, overall hospital admission ( $28.5 \%$ vs. $82.6 \%, P<0.001)$, intensive care unit admission (1.7\% vs. $37.6 \%, P<0.001)$, death after admission $(1.4 \%$ vs. $10.3 \%$, $\mathrm{P}=0.034)$, and total mortality ( $0.7 \%$ vs. $9.2 \%, \mathrm{P}<0.001)$. The odds ratios (ORs) for injury severity were as follows: age 70 years or older ( $\mathrm{OR}, 2.124 ; 95 \%$ confidence interval $[\mathrm{Cl}], 1.239-3.642)$, head injury $(\mathrm{OR}, 10.441 ; 95 \% \mathrm{Cl}, 5.465-19.950)$, and torso injury $(\mathrm{OR}, 4.858 ; 95 \% \mathrm{Cl}, 2.495$ 9.458).

Conclusion The proportions of patients aged 70 years or older, head and torso injuries, injuries from traffic accidents and falls, and injuries in the daytime were higher in the severe group. Our results highlight the need for measures to address these factors to lower the incidence of severe injuries.

Keywords Wheelchairs; Equipment and supplies; Aged; Risk factors; Wounds and injuries
eISSN: 2383-4625

Received: 22 June 2020

Revised: 13 August 2020

Accepted: 14 August 2020

Correspondence to: Jae-Hyug Woo Department of Emergency and Critical Care Medicine, Gachon University Gil Medical Center, Gachon University College of Medicine, 783 Namdongdaero, Namdong-gu, Incheon 21556, Korea

E-mail:emmetalkiller@gilhospital.com ORCID https://orcid.org/0000-0001-9710-3265

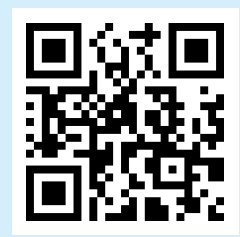

How to cite this article:

Choi SW, Woo JH, Hyun SY, Jang JH, Choi WS. Factors associated with injury severity among users of powered mobility devices. Clin Exp Emerg Med 2021;8(2):103-110. https://doi.org/10.15441/ceem.20.078

This is an Open Access article distributed under the terms of the Creative Commons Attribution Non-Commercial License (https:// creativecommons.org/licenses/by-nc/4.0/). 


Capsule
What is already known
Past studies have shown that most injuries associated with wheelchairs result from tips and falls from wheelchairs,
with the most common injuries including fracture, contusion, and laceration of extremities, while severe injuries are
rare. However, the inherent features of injuries from powered mobility devices are not well studied.
$\begin{aligned} & \text { What is new in the current study } \\ & \text { Factors associated with severe injuries due to the use of powered mobility devices are patients aged } 70 \text { years or older, } \\ & \text { head and torso injuries, injury from traffic accidents and falls, and injuries in the daytime. }\end{aligned}$

\section{INTRODUCTION}

Wheelchairs are an important means by which older people and people with disabilities who have reduced mobility can continue participating in the activities of daily living and engaging in social activities through the enablement of independent transportation. ${ }^{1,2}$ While manual wheelchairs serve this purpose, powered wheelchairs and four-wheel mobility scooters, both of which are referred to as "powered mobility devices", are now beginning to replace them.-5 According to the "current needs and use of major assistive devices for people with disabilities", as published by Statistics Korea, the number of powered mobility device users had increased from 22,000 in 2005 to 112,000 in $2014 .{ }^{6}$ As older adults are now more frequently utilizing powered mobility devices as a means of transport, the actual number of powered mobility device users is estimated to be higher than that reported. ${ }^{2,7}$

Previous studies that examined the features of injuries among wheelchair users reported that a majority of injuries result from tips and falls from wheelchairs, with fracture, contusion, and laceration of the extremities the most commonly cited injuries, and that severe injuries occur rarely. ${ }^{4,58-11}$ However, those studies are limited in that they only examined the overall features without distinguishing between manual wheelchairs, powered wheelchairs, and four-wheel mobility scooters, thereby failing to identify the unique features of injuries sustained from the use of powered mobility devices. Although a recent study reported on the injuries resulting from the use of powered mobility devices, there is a lack of sufficient data on the features of these injuries. ${ }^{12}$

Accordingly, this study aimed to identify the features of powered mobility device-related injuries and identify the predictors of injury severity through comparisons of the features of patients with mild/moderate injuries and those with severe injuries, and ultimately explore measures aimed at the prevention of injuries and lowering the injury severity.

\section{METHODS}

\section{Ethics statement}

This study was performed in accordance with the Declaration of Helsinki and was approved by the institutional review board of Gachon University Gil Medical Center (GBIRB2020-162). The requirement for informed consent was waived due to the retrospective nature of the study.

\section{Data source}

This study used data from the Emergency Department-based Injury In-depth Surveillance (EDIIS), which were prospectively collected from across 23 hospitals nationwide from 2011 to 2018 by the Korea Centers for Disease Control and Prevention. The EDIIS was established for the collection of information on the mechanism and cause of injuries among patients admitted to an emergency department (ED) and utilizes this information for the development of injury prevention and management policies. As of 2020, the EDs of 23 nationwide hospitals are participating in this surveillance. When a patient with an injury presents to the ED, healthcare providers, such as physicians, nurses, and emergency medical technicians, first encode the injury-related information in a computer. A trained coordinator then collects the additional information based on the emergency care activity log, ED records, nursing records, and admission and discharge records and registers them in the comprehensive disease management system (http://is.cdc.go.kr) along with the information collected in the ED. The registered data are reviewed by the quality control team at the Korea Centers for Disease Control and Prevention, which provides feedback on errors, unknown data, and missing data to the hospital. ${ }^{13}$

\section{Inclusion and exclusion criteria}

We used data pertaining to powered wheelchair or four-wheel mobility scooter-related injuries that occurred between 2011 and 
2018 in our analysis. To this end, we initially extracted data entered using the code " $\mathrm{C} 1.98 .05$ (powered wheelchair)" or "C1.98.98 (other specified ground transportation [including scooters for people with disabilities])" for the cause of injury and data with injury descriptions including "powered wheelchair" and "four-wheel mobility scooter." Data that were not pertinent to injuries sustained through the use of powered mobility device, data on vehicles other than powered mobility devices (such as cars for people with disabilities or carts for those with a handicap), data with missing information on whether the users of the scooters were elderly people or those with a disability, duplicate data, and missing data on the excess mortality ratio-adjusted injury severity score (EMR-ISS) were excluded.

\section{Collected variables and definition of terms}

Of the injury-related data, general parameters pertaining to the injury, including patients' sex, age, time, activity and alcohol intake status at time of the injury, location at which the injury was sustained (indoor vs. outdoor), detailed accident location, cause of crash, and injury mechanism, and parameters related to the injury outcomes, including the vital signs at the ED visit, Glasgow Coma Scale, surgery, severity (EMR-ISS), ED outcomes, and admission outcomes, were used in the analysis.

The accident location was simply classified as "indoor" and "outdoor" and more specifically as "residence" (home, nursing home, etc.), "roadway", "sidewalk", "other facilities where vehicles can be driven" (parking lot, mart, park, etc.), and "others". Cause of crash classified into "motor vehicles" such as cars and motorcycles and "fixed objects" such as floors, walls, and stairs.

The injury mechanisms were reclassified as follows, based on the coded data and specific descriptions pertaining to the injury: tips and falls, user injury caused by tipping and falling of the powered mobility device; traffic accidents, injury caused by collision with a moving car or motorcycle; collisions, injury caused by collision with a stationary object; and falls, injury caused by falling on a slope with a different height.

The EMR-ISS is a scale used for the assessment of the severity of an injury based on the International Classification of Diseases 10th edition (ICD-10). ${ }^{14}$ Excess mortality ratio is calculated as the percentage of deaths among patients diagnosed with each ICD10 code against the expected mortality in the general population. The EMR-ISS is calculated by the summation of the squares of the three highest EMR grades from all the ICD-10 codes of a particular patient: EMR-ISS $=$ (first highest EMR grade $)^{2}+($ second high-

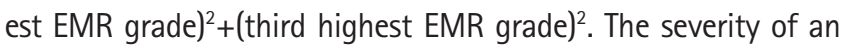
injury can be classified into four groups depending on the EMRISS: mild ( $1<$ EMR-ISS $\leq 8)$, moderate $(9<$ EMR-ISS $\leq 24)$, se- vere $(25<$ EMR-ISS $\leq 74)$, and critical (EMR-ISS $=75$ or death). The EMR-ISS can relatively accurately predict mortality and is known to have a stronger discriminatory power than the more widely used ICD-9-based injury severity score. In this study, we divided patients into the following groups based on the severity of their injury, as assessed using the EMR-ISS: mild/moderate group $(<25)$ and severe group $(\geq 25) \cdot{ }^{12,14}$

\section{Primary outcomes}

The primary outcomes of this study were the comparison of the injury-related features and treatment outcomes between the mild/ moderate and severe groups and identification of the predictors of injury severity.

\section{Statistical analysis}

Data were analyzed using PASW Statistics ver. 18.0 (SPSS Inc., Chicago, IL, USA). Continuous variables are expressed as median and interquartile ranges, and comparisons were performed using the Mann-Whitney U-test. Categorical variables are expressed as frequencies and percentages, and comparisons were performed using the chi-square test or Fisher exact test. Multivariate binary logistic regression analysis was used to assess the independent predictors of injury severity. All variables with a significance level $<0.10$ in the univariate analysis were included in the multivariate logistic regression analysis. Variables with collinearity were excluded. The backward stepwise method was used to select the final model. The Hosmer-Lemeshow test was used for the assessment of the goodness of fit. All statistical tests were two-sided, and a P-value of $<0.05$ was considered significant.

\section{RESULTS}

\section{Baseline characteristics of the study population}

Of the 487 patients with injuries related to the use of powered mobility devices, data on 80 (of which 49 did not involve an injury sustained by the driver of the powered mobility device, four in which vehicles other than powered mobility devices were used [such as cars for people with disabilities or carts for those with a handicap], 13 cases in which it was undetermined whether the users of the scooters were elderly or had a disability, six cases in which duplicate data were present, and eight cases in which EMRISS data were missing) were excluded. Data on only 407 people were included in this study (Fig. 1). In total, 298 (73.2\%) patients were assigned to the mild/moderate group and 109 (26.8\%) to the severe group.

Table 1 summarizes the baseline injury-related characteristics of the mild/moderate and severe groups. Both groups had a higher 


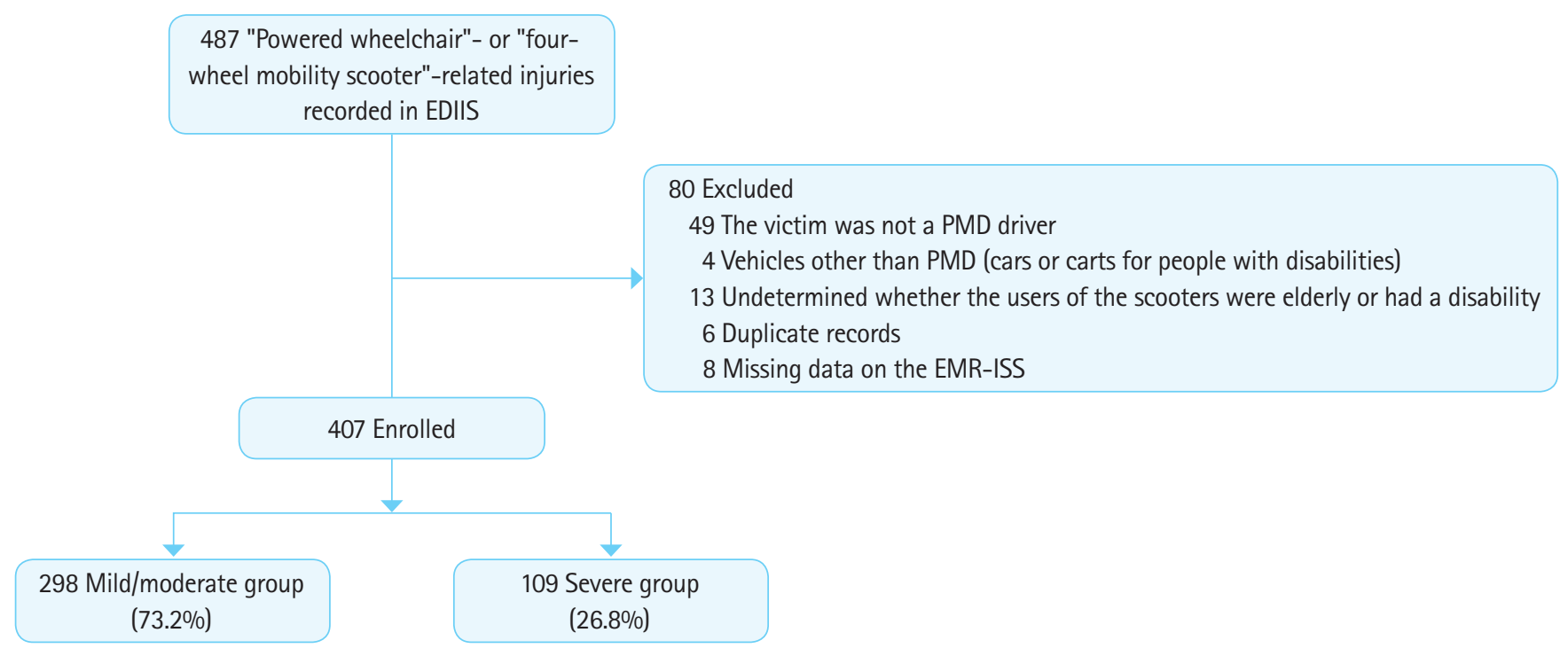

Fig. 1. Flowchart of the patient selection process. EDIIS, Emergency Department-based Injury In-depth Surveillance; PMD, powered mobility device; EMR-ISS, excess mortality ratio-adjusted injury severity score.

Table 1. General characteristics of the study population and the injury mechanisms

\begin{tabular}{|c|c|c|c|}
\hline Variable & $\begin{array}{l}\text { Mild/moderate } \\
\text { group }(n=298)\end{array}$ & $\begin{array}{l}\text { Severe group } \\
\quad(n=109)\end{array}$ & P-value \\
\hline Sex, male & $183(61.4)$ & $69(63.3)$ & 0.728 \\
\hline Age (yr) & $67.0(53.0-76.0)$ & $73.0(62.0-79.5)$ & $<0.001$ \\
\hline $\mathrm{Age}_{1} \geq 70 \mathrm{yr}$ & $128(43.0)$ & 65 (59.6) & 0.003 \\
\hline Time (9 a.m. to 6 p.m.) & $215(72.6)$ & $89(82.4)$ & 0.044 \\
\hline Season & & & 0.536 \\
\hline Spring & $81(27.2)$ & $26(23.9)$ & \\
\hline Summer & $80(26.8)$ & $35(32.1)$ & \\
\hline Autumn & $86(28.9)$ & $34(31.2)$ & \\
\hline Winter & $51(17.1)$ & $14(12.8)$ & \\
\hline Drunk driving & $20(6.7)$ & $7(6.4)$ & 0.887 \\
\hline Purpose of driving, working & $69(23.2)$ & $21(19.3)$ & 0.403 \\
\hline Accident location, outdoor & 279 (93.6) & $103(94.5)$ & 0.916 \\
\hline Detailed accident location & & & 0.353 \\
\hline Residence & $21(7.0)$ & $5(4.6)$ & \\
\hline Roadway & $194(65.1)$ & $71(65.1)$ & \\
\hline Sidewalk & $59(19.8)$ & $18(16.5)$ & \\
\hline $\begin{array}{l}\text { Other facilities where } \\
\text { vehicles can be driven }\end{array}$ & $7(2.3)$ & $3(2.8)$ & \\
\hline Others & $17(5.7)$ & $12(11.0)$ & \\
\hline Crash opponents & & & 0.381 \\
\hline Motor vehicles & $88(31.3)$ & $36(36.0)$ & \\
\hline Fixed objects & $184(65.5)$ & $63(63.0)$ & \\
\hline Others & $9(3.2)$ & $1(1.0)$ & \\
\hline Mechanism of injury & & & 0.042 \\
\hline Tips and falls & $141(51.8)$ & $40(40.8)$ & \\
\hline Traffic accidents & $88(32.4)$ & $36(36.7)$ & \\
\hline Collisions & $10(3.7)$ & $1(1.0)$ & \\
\hline Falls & 33 (12.1) & 21 (21.4) & \\
\hline
\end{tabular}

Values are presented as number (\%) or median (interquartile range).

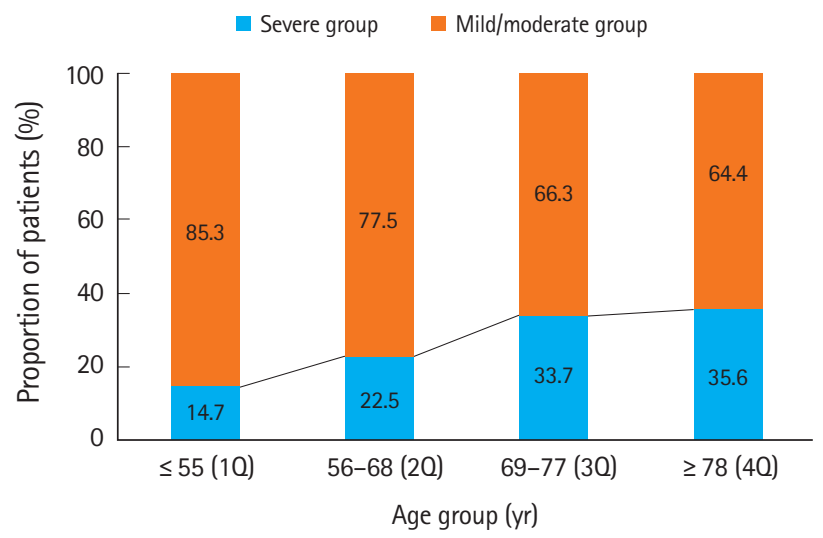

Fig. 2. Relationship between injury severity and age group $(\mathrm{P}=0.001)$. 0 , quartile.

percentage of male patients, and no significant differences were observed between the two groups in terms of sex (61.4\% vs. 63.3\%, $P=0.728$ ). The median age in the severe group was significantly higher than that in the mild/moderate group (67.0 [53.0-76.0] vs. 73.0 [62.0-79.5], $\mathrm{P}<0.001$ ). In addition, the severe group had a significantly higher percentage of patients aged 70 years or older. When patients were divided into quartiles, the injury severity significantly increased with increasing age (Fig. 2) $(P=0.001)$. The severe group had a higher percentage of daytime injuries $(72.6 \%$ vs. $82.4 \%, P=0.044)$ and injuries caused by traffic accidents and falls $(P=0.042)$.

\section{Outcomes of the study population}

Table 2 presents a summary of the injury-related outcomes in each group. The severe group had a significantly higher percent- 
Table 2. Comparison of patient between the mild/moderate and severe groups

\begin{tabular}{lccc}
\hline Variable & Mild/moderate group $(\mathrm{n}=298)$ & Severe group $(\mathrm{n}=109)$ & P-value \\
\hline Systolic blood pressure $(\mathrm{mmHg})$ & $136.0(120.0-155.3)$ & $134.5(120.0-153.8)$ & 0.915 \\
Diastolic blood pressure $(\mathrm{mmHg})$ & $80.0(70.0-90.0)$ & $80.0(70.0-90.0)$ & 0.598 \\
Heart rate $(\mathrm{min})$ & $81.0(72.0-92.0)$ & $83.0(73.0-96.8)$ & 0.336 \\
Respiration rate $(\mathrm{min})$ & $20.0(18.0-20.0)$ & $20.0(18.0-20.0)$ & 0.043 \\
Body temperature $\left({ }^{\circ} \mathrm{C}\right)$ & $36.6(36.3-36.8)$ & $36.5(36.2-36.8)$ & 0.132 \\
Glasgow Coma Scale score & $15.0(15.0-15.0)$ & $15.0(15.0-15.0)$ & $<0.001$ \\
Head injury & $115(38.6)$ & $88(80.7)$ & $<0.001$ \\
Spine injury & $32(10.7)$ & $15(13.8)$ & 0.398 \\
Torso injury & $50(16.8)$ & $35(32.1)$ & 0.001 \\
Upper extremities injury & $63(21.1)$ & $24(22.0)$ & 0.848 \\
Lower extremities injury & $99(33.2)$ & $12(11.0)$ & $<0.001$ \\
Surgery & $40(13.4)$ & $15(13.8)$ & 0.929 \\
Admission & $85(28.5)$ & $90(82.6)$ & $<0.001$ \\
ICU admission & $5(1.7)$ & $41(37.6)$ & $<0.001$ \\
Death in ED & $1(0.3)$ & $2(1.8)$ & 0.176 \\
Death after admission & $1(1.4)$ & $8(10.3)$ & 0.034 \\
Death (overall) & $2(0.7)$ & $10(9.2)$ & $<0.001$ \\
\hline
\end{tabular}

Values are presented as median (interquartile range) or number (percentage).

$I C U$, intensive care unit; $E D$, emergency department.

Table 3. Multivariate logistic regression analysis of the independent factors associated with powered mobility device-related injury severity

\begin{tabular}{lccc}
\hline \multirow{2}{*}{ Variable } & Odds ratio $^{\text {a) }}$ & \multicolumn{2}{c}{$95 \%$ confidence interval } \\
\cline { 3 - 4 } & & Lower & Upper \\
\hline Sex, male & 1.370 & 0.788 & 2.384 \\
Age, $\geq 70 \mathrm{yr}$ & 2.124 & 1.239 & 3.642 \\
Head injury & 10.441 & 5.465 & 19.950 \\
Torso injury & 4.858 & 2.495 & 9.458 \\
\hline
\end{tabular}

Variables included in this analysis were sex, age ( $\geq 70 \mathrm{yr}$ ), head injuries, torso injuries, and injury mechanism (falls).

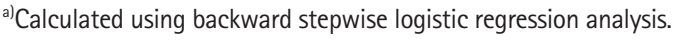

age of head and torso injuries (38.6\% vs. 80.7\%, $P<0.001 ; 16.8 \%$ vs. $32.1 \%, P=0.001)$, while the mild/moderate group had a higher percentage of lower limb injuries (33.2\% vs. $11.0 \%, \mathrm{P}<0.001)$. The severe group had a higher rate of overall admission and intensive care unit admission (28.5\% vs. $82.6 \%, \mathrm{P}<0.001 ; 1.7 \%$ vs. $37.6 \%, P<0.001)$, as well as death after admission and total mortality ( $1.4 \%$ vs. $10.3 \%, \mathrm{P}=0.034 ; 0.7 \%$ vs. $9.2 \%, \mathrm{P}<0.001)$.

\section{Multivariate logistic regression analysis}

The results of the multivariate logistic regression analysis focusing on the severity of the injuries sustained from the use of powered mobility devices showed that severe injury was significantly associated with older age (70 years or older) (odds ratio [OR], 2.124; 95\% confidence interval [Cl], 1.239-3.642), head injury (OR, 10.441; $95 \% \mathrm{Cl}, 5.465-19.950)$, and torso injury $(\mathrm{OR}, 4.858 ; 95 \% \mathrm{Cl}, 2.495-$ 9.458) (Table 3).

\section{DISCUSSION}

In the present study, the proportions of patients aged 70 years or older, head and torso injuries, injuries from traffic accidents and falls, and injuries sustained in the daytime were higher in the severe group. It is necessary to examine the background and cause of severe injuries by each of these factors and to explore measures to prevent severe injuries based on such examination.

\section{Age}

Age influences the quality of sensory reception and processing in response to external stimuli and motor capabilities. The deterioration of the perceptual and cognitive abilities required for driving based on visual information increases the driver's exposure to risky situations that may lead to an accident. ${ }^{15}$ Further, aging weakens balance and strength performance, which in turn diminishes one's torso control ability; this is speculated to increase the risk of secondary injury by easily pushing the user out of the device upon impact. ${ }^{16}$ As aging is an uncorrectable factor, measures to lower the incidence of accidents are needed in order to decrease the incidence of severe injuries among older adult users. To this end, environmental factors that may trigger an accident as well as users' level of awareness on safe driving need to be improved; in particular, older adult users should be informed about the basic manipulation, safe use, and regular maintenance and repair of powered mobility devices..$^{15,17,18}$ The degeneration of sensory and motor functions accompanying neurological and musculoskeletal disorders that develop with advancing age may have an impact 
on disease severity. However, we could not determine the correlation between the presence of disease and injury severity due to the lack of information on comorbidities in our data. Therefore, additional studies should be conducted to examine whether age and the presence of underlying disease are independent variables that are correlated with injury severity.

\section{Head and torso injuries}

The higher percentage of head and torso injuries observed in the severe group may be attributed to the fact that the injured areas tend to include body parts that are essential for the maintenance of stable vital signs, such as the brain and internal organs. Therefore, the use of protective devices for the head and torso may be effective in lowering the incidence of severe injuries. Helmets are known to reduce the incidence and severity of brain damage from accidents involving mobility devices used in a similar environment, such as bicycles and motorcycles. ${ }^{19,20}$ Furthermore, the use of seat belts lowers the incidence and severity of injury by reducing the possibility of secondary injury and preventing users from being pushed off the mobility device upon impact. ${ }^{21-23}$ According to the "Ministry of Food and Drug Safety Notification 2019-61, Standard specifications for medical devices, Schedule 2. '48. Powered wheelchairs and medical scooters", seat belts are not mandatory when driving a powered mobility device. ${ }^{24}$ In fact, a survey conducted among Korean users reported that more than $70 \%$ of users do not wear a seat belt. ${ }^{4}$ To reduce the severity of injuries, the use of protective devices such as helmets and seat belts should be strongly recommended.

\section{Traffic accidents}

According to the Road Traffic Act, powered mobility device users are considered pedestrians and these devices are only allowed to be operated on sidewalks. ${ }^{25}$ However, the uneven, cracked, and sunken surfaces of some block-paved sidewalks, presence of trees lining the street and standing signboards, and certain road facilities may hinder the use of powered mobility devices, causing accidents. ${ }^{5,26}$ Due to these factors, users operate powered mobility devices on the road instead of the sidewalk, and consequent collisions with cars and motorcycles lead to severe injuries. To prevent such injuries, sidewalks should be altered to enable easy powered mobility device use, and users should be educated about traffic laws and safe driving. Furthermore, it may be helpful to install reflectors or lights on powered mobility devices to allow for automobile drivers to easily detect them. While we were unable to obtain official statistics pertaining to traffic accidents involving powered mobility devices in South Korea, our data analysis revealed that traffic accidents account for $30.5 \%$ of all inju- ries, and that the percentage of injuries caused by traffic accidents increases every year, having risen from 10.5\% in 2011 to $24.2 \%$ in 2014 and $35.0 \%$ in 2018. Recently, the illegal driving of powered mobility devices on the road and consequent traffic accidents have emerged as social problems. In addition to performing an accurate statistics survey on relevant accidents, future studies should compare the injury-related features and outcomes with those related to the use of other similar modes of transport, such as bicycles and motorcycles, as well as those among pedestrians.

\section{Falls}

Compared to other types of injuries, falls involve a greater impact; this may be the reason for the higher percentage of falls in the severe group. A detailed analysis of our data revealed that falls generally occur on stairs (29.1\%) and farm roads (25.5\%). A previous study found that stairs are associated with fatal wheelchairrelated injuries. ${ }^{27}$ These results highlight the need to expand the proportion of accessible facilities such as entryways, lifts, or elevators for powered mobility devices. As most farm roads are not paved, roads with uneven surfaces and unclear boundaries between a level ground and steep slope potentially cause accidents. Particularly, farm roads are frequently utilized by older adult users in agricultural areas; hence, it is necessary to maintain these roads to make them powered mobility device-friendly and reduce the severity of any injuries.

\section{Daytime injuries}

A previous study observed that the rate of severe injuries is high among male patients between the ages of 6 and 17 years, which is a period marked by a high level of involvement in outdoor sports and social activities. ${ }^{8}$ The high percentage of daytime injuries in the severe group in our study is also believed to be due to the fact that occupational activities, routine daily activities, and leisure activities are frequently performed during the day. Furthermore, a substantial percentage of the severe injuries observed in association with traffic accidents may be due to the presence of higher levels of traffic during the day.

This study has some limitations. First, we used data collected nationwide during a specific period; the retrospective analysis of this data may be susceptible to patient selection bias. Second, the data used in this study were collected from 23 large hospitals nationwide, and hence, the overall severity of the injuries and incidence of injury mechanisms correlated with severe injuries may have been overestimated. Third, multiple terms were used to refer to powered mobility devices, such as powered wheelchairs, scooters for people with disabilities, electric mobility scooters, and four- 
wheel mobility scooters, in the EDIIS; therefore, some data may have been omitted in the search.

In summary, the percentages of patients aged 70 years or older, head and torso injuries, injuries from traffic accident and falls, and injuries sustained during the day were higher in the group of patients with severe injuries in association with powered mobility device use. To lower the rate of severe injuries, it is important to provide education on basic device manipulation, safe driving, regular maintenance and repair, and traffic laws; to strongly recommend the use of protective devices such as helmets and seat belts; and to improve the conditions of frequently used sidewalks and farm roads to be powered mobility device-friendly.

\section{CONFLICT OF INTEREST}

No potential conflict of interest relevant to this article was reported.

\section{ACKNOWLEDGMENTS}

The data used in this study were provided by the Korea Centers for Disease Control and Prevention.

\section{REFERENCES}

1. Pettersson I, Tornquist K, Ahlstrom G. The effect of an outdoor powered wheelchair on activity and participation in users with stroke. Disabil Rehabil Assist Technol 2006;1:235-43.

2. Brandt A, Iwarsson S, Stahle A. Older people's use of powered wheelchairs for activity and participation. J Rehabil Med 2004; 36:70-7.

3. Byeon YC, Kim SH, Yoon SY, et al. National survey on persons with disabilities 2008. Seoul: Ministry of Health and Welfare; 2009.

4. Kong JY. The safety incidents survey of mobility assistive device users. J Spec Educ Rehabil Sci 2013;52:1-16.

5. Lee ST. Survey of wheelchair safety accidents. Eumseong: Korea Consumer Agency; 2011.

6. Statistics Korea. Current needs and use of major assistive devices for people with disabilities [Internet]. Daejeon: Statistics Korea; 2018 [cited 2020 Jun 14]. Available from: http://kosis. $\mathrm{kr} / \mathrm{statHtml} / \mathrm{statHtml}$. do? mode $=$ tab\&torgld $=117 \& \mathrm{ttblld}=\mathrm{DT}_{-}$ 11732S0113\&tvw_cd =.

7. Evans S, Frank AO, Neophytou C, de Souza L. Older adults' use of, and satisfaction with, electric powered indoor/outdoor wheelchairs. Age Ageing 2007;36:431-5.

8. Xiang $H$, Chany AM, Smith GA. Wheelchair related injuries treated in US emergency departments. Inj Prev 2006;12:8-11.

9. Chen WY, Jang Y, Wang JD, et al. Wheelchair-related accidents: relationship with wheelchair-using behavior in active community wheelchair users. Arch Phys Med Rehabil 2011;92:892-8.

10. Nelson AL, Groer $S$, Palacios $P$, et al. Wheelchair-related falls in veterans with spinal cord injury residing in the community: a prospective cohort study. Arch Phys Med Rehabil 2010;91: 1166-73.

11. Gaal RP, Rebholtz N, Hotchkiss RD, Pfaelzer PF. Wheelchair rider injuries: causes and consequences for wheelchair design and selection. J Rehabil Res Dev 1997;34:58-71.

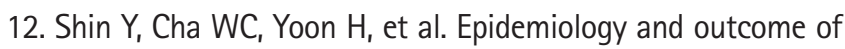
powered mobility device-related injuries in Korea. J Korean Med Sci 2020;35:e60.

13. Choi ES, Jang JH, Woo JH, Choi JU, Cho JS, Yang HJ. Pediatric trampoline-related injuries in a nationwide registry in South Korea, 2011 to 2016. Yonsei Med J 2018;59:989-94.

14. Kim J, Shin SD, Im TH, et al. Development and validation of the Excess Mortality Ratio-adjusted Injury Severity Score using the International Classification of Diseases 10th edition. Acad Emerg Med 2009;16:454-64.

15. Boot WR, Stothart C, Charness N. Improving the safety of aging road users: a mini-review. Gerontology 2014;60:90-6.

16. Granacher U, Gollhofer A, Hortobagyi T, Kressig RW, Muehlbauer T. The importance of trunk muscle strength for balance, functional performance, and fall prevention in seniors: a systematic review. Sports Med 2013;43:627-41.

17. Hansen R, Tresse $S$, Gunnarsson RK. Fewer accidents and better maintenance with active wheelchair check-ups: a randomized controlled clinical trial. Clin Rehabil 2004;18:631-9.

18. McClure LA, Boninger ML, Oyster ML, et al. Wheelchair repairs, breakdown, and adverse consequences for people with traumatic spinal cord injury. Arch Phys Med Rehabil 2009;90: 2034-8.

19. Attewell RG, Glase K, McFadden M. Bicycle helmet efficacy: a meta-analysis. Accid Anal Prev 2001;33:345-52.

20. Liu BC, Ivers R, Norton R, Boufous $S$, Blows $S$, Lo SK. Helmets for preventing injury in motorcycle riders. Cochrane Database Syst Rev 2008;(1):CD004333.

21. Corfman TA, Cooper RA, Fitzgerald SG, Cooper R. Tips and falls during electric-powered wheelchair driving: effects of seatbelt use, legrests, and driving speed. Arch Phys Med Rehabil 2003:84:1797-802.

22. Cooper RA, Dvorznak MJ, O'Connor TJ, Boninger ML, Jones DK. Braking electric-powered wheelchairs: effect of braking method, seatbelt, and legrests. Arch Phys Med Rehabil 1998; 79:1244-9. 
23. Fast $A$, Sosner J, Begeman P, Thomas M, Drukman D. Forces, moments, and acceleration acting on a restrained dummy during simulation of three possible accidents involving a wheelchair negotiating a curb: comparison between lap belt and four-point belt. Am J Phys Med Rehabil 1997;76:370-7.

24. Ministry of Food and Drug Safety. Standard specifications for medical devices (notification 2019-61) [Internet]. Cheongju: Ministry of Food and Drug Safety; 2019 [cited 2020 Jun 14]. Available from: https://mfds.go.kr/brd/m_211/view.do?seq = 14345.
25. Road Traffic Act No. 17891, Article 2, Paragraph 17 (Dec 31, 1961; modified Jun 9, 2020).

26. Gavin-Dreschnack D, Nelson A, Fitzgerald S, et al. Wheelchairrelated falls: current evidence and directions for improved quality care. J Nurs Care Qual 2005;20:119-27.

27. Calder CJ, Kirby RL. Fatal wheelchair-related accidents in the United States. Am J Phys Med Rehabil 1990;69:184-90. 